\title{
Superexponential stabilizability of evolution equations of parabolic type via bilinear control
}

\author{
Fatiha Alabau- Boussouira, Piermarco Cannarsa and Cristina Urbani(i)
}

Abstract. We study the stabilizability of a class of abstract parabolic equations of the form

$$
u^{\prime}(t)+A u(t)+p(t) B u(t)=0, \quad t \geq 0
$$

where the control $p(\cdot)$ is a scalar function, $A$ is a self-adjoint operator on a Hilbert space $X$ that satisfies

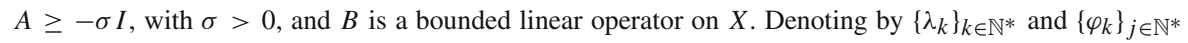
the eigenvalues and the eigenfunctions of $A$, we show that the above system is locally stabilizable to the eigensolutions $\psi_{j}=\mathrm{e}^{-\lambda_{j} t} \varphi_{j}$ with doubly exponential rate of convergence, provided that the associated linearized system is null controllable. Moreover, we give sufficient conditions for the pair $\{A, B\}$ to satisfy such a property, namely a gap condition for $A$ and a rank condition for $B$ in the direction $\varphi_{j}$. We give several applications of our result to different kinds of parabolic equations.

\section{Introduction}

In the field of control theory of dynamical systems, a huge amount of works is devoted to the study of models in which the control enters as an additive term (boundary or internal locally distributed controls); see, for instance, the books [22,23] by Lions. On the other hand, these kinds of control systems are not suitable to describe processes that change their physical characteristics in response to the control. This issue is quite common for the so-called smart materials and in many biomedical, chemical and nuclear chain reactions. Indeed, under the process of catalysis some materials are able to change their principal parameters (see the examples in the monograph [20] by Khapalov for more details.)

To deal with these situations, an important role is played by multiplicative controls, that is, controls that appear in the equations as coefficients.

Mathematics Subject Classification: 35Q93, 93C25, 93C10, 35K10

Keywords: Bilinear control, Stabilization, Evolution equations, Moment method.

This paper was partly supported by the INdAM National Group for Mathematical Analysis, Probability and their Applications. P. Cannarsa: This author acknowledges support from the MIUR Excellence Department Project awarded to the Department of Mathematics, University of Rome Tor Vergata, CUP E83C18000100006. C. Urbani: This author is grateful to University Italo Francese (Vinci Project 2018). 
Due to a weaker control action, exact controllability results are not to be expected with multiplicative controls. Nevertheless, approximate controllability has been obtained for different types of initial/target conditions. For instance, in [18] Khapalov proved a result of nonnegative approximate controllability for a $1 D$ semilinear parabolic equation. In [19], the same author proved approximate and exact null controllability for a bilinear parabolic system with reaction term satisfying Newton's law. Paper [16], by Floridia, is devoted to the study of global approximate multiplicative controllability for nonlinear degenerate parabolic problems. In $[8,10]$, results of approximate controllability of a one-dimensional reaction-diffusion equation via multiplicative control and with sign changing data are proved. Finally, we recall that some results of approximate controllability have been also obtained for hyperbolic equations with bilinear control (see, for instance, [13]).

An even more specific and weaker class of controls are bilinear controls which enter the equation as scalar functions of time as, for instance, in the following system:

$$
\left\{\begin{array}{l}
u^{\prime}(t)+A u(t)+p(t) B u(t)=0, \quad t>0 \\
u(0)=u_{0}
\end{array}\right.
$$

Here, $A: D(A) \subset X \rightarrow X$ is a linear operator on a Hilbert space $(X,\langle\cdot, \cdot\rangle)$ and $B$ is a bounded linear operator on $X$.

A fundamental result in control theory for this type of evolution equations is the one due to Ball et al. [3] which establishes that under the assumption that $A$ is the generator of a $C^{0}$-semigroup of bounded linear operators on $X$, system (1) fails to be controllable. Indeed, denoting by $u\left(t ; p, u_{0}\right)$ the unique solution of $(1)$, it is shown in [3] that the attainable set from $u_{0}$, defined by,

$$
S\left(u_{0}\right)=\left\{u\left(t ; p, u_{0}\right) ; t \geq 0, p \in L_{\mathrm{loc}}^{r}([0,+\infty), \mathbb{R}), r>1\right\}
$$

has a dense complement.

On the other hand, when $B$ is unbounded, the possibility of proving a positive controllability result remains open. This idea of exploiting the unboundedness of $B$ was used by Beauchard and Laurent in [5] to study the Schrödinger equation:

$$
\left\{\begin{array}{l}
i u_{t}(t, x)+u_{x x}(t, x)+p(t) \mu(x) u(t, x)=0, \quad(t, x) \in(0, T) \times(0,1) \\
u(t, 0)=u(t, 1)=0
\end{array}\right.
$$

For such an equation, the authors proved local exact controllability around the ground state in a stronger topology than the natural one of $X=H^{2} \cap H_{0}^{1}(0,1)$, for which the multiplication operator $B u(t, x)=\mu(x) u(t, x)$ is unbounded. In other terms, the above result could be regarded as a description of the attainable set from the ground state as a certain submanifold of the original Banach space.

Following the same strategy, Beauchard [4] studied the controllability of the wave equation:

$$
\left\{\begin{array}{l}
u_{t t}(t, x)-u_{x x}(t, x)-p(t) \mu(x) u(t, x)=0, \quad(t, x) \in(0, T) \times(0,1) \\
u_{x}(t, 0)=u_{x}(t, 1)=0 .
\end{array}\right.
$$


In both papers [4,5], a key point of the analysis is the application of the inverse mapping theorem, which is made possible by the controllability of the associated linearized problem. This is the reason why, for parabolic equations, the above strategy meets an obstruction: The spaces for which one can prove controllability of the linearized equation are not well adapted to the use of the inverse map technique.

It is important to stress the fact that the negative result by Ball, Marsden and Slemrod still allows for exact controllability to a trajectory $\bar{u}\left(\cdot ; \bar{p}, \overline{u_{0}}\right)$ by a bilinear control. With respect to controllability to trajectories with additive controls, we mention the result in [15] by Fernández-Cara, Guerrero, Imanuvilov and Puel for the Navier-Stokes equations.

In this paper, we are interested in studying the possibility of steering the solution of (1), with a bilinear control, to any eigensolution of the operator $A$. In order to explain what an eigensolution is, let $X$ be a separable Hilbert space, $A: D(A) \subset X \rightarrow X$ be a self-adjoint operator with compact resolvent, satisfying $A \geq-\sigma I$, with $\sigma \geq 0$ (see Sect. 2 for more on notation and assumptions) and let $\left\{\lambda_{k}\right\}_{k \in \mathbb{N}^{*}}$ be the eigenvalues of $A,\left(\lambda_{k} \leq \lambda_{k+1}, \forall k \in \mathbb{N}^{*}\right)$, with associated eigenfunctions $\left\{\varphi_{k}\right\}_{k \in \mathbb{N}^{*}}$. For every $j \geq 1$, the function $\psi_{j}(t)=\mathrm{e}^{-\lambda_{j} t} \varphi_{j}$ will be called the $j$ th eigensolution of $A$.

Our approach is based on the null controllability in some time $T>0$ of the linearized system:

$$
\left\{\begin{array}{l}
y^{\prime}(t)+A y(t)+p(t) B \varphi_{j}=0, \\
y(0)=y_{0}
\end{array}\right.
$$

a property that we call $j$-null controllability of the pair $\{A, B\}$.

Indeed, in Theorem 3.7 we show that, if $\{A, B\}$ is $j$-null controllable, then the nonlinear system (1) is locally superexponentially stabilizable to the eigensolution $\psi_{j}$ of $A$. The proof relies on a "quadratic estimate" for the difference between the solution of (1) and its linearization along $\psi_{j}$ (Proposition 4.4).

Finally, we investigate the notion of $j$-null controllability, providing sufficient conditions for such a property. In Theorem 3.8, we prove that $\{A, B\}$ is $j$-null controllable if the gap condition

$$
\sqrt{\lambda_{k+1}-\lambda_{1}}-\sqrt{\lambda_{k}-\lambda_{1}} \geq \alpha, \quad \forall k \in \mathbb{N}^{*}
$$

holds true for some $\alpha>0$, and $B$ satisfies

$$
\begin{aligned}
& \text { (i) }\left\langle B \varphi_{j}, \varphi_{k}\right\rangle \neq 0, \quad \forall k \in \mathbb{N}^{*}, \\
& \text { (ii) } \exists \tau>0: \sum_{k \in \mathbb{N}^{*}} \frac{\mathrm{e}^{-2 \lambda_{k} \tau}}{\left|\left\langle B \varphi_{j}, \varphi_{k}\right\rangle\right|^{2}}<+\infty
\end{aligned}
$$

We recall that condition (4), which is required to use the moment method (see, e.g., $[4,5])$, is usually satisfied by elliptic operators in one space dimension. As far as (5) is concerned, we observe that (3) fails to be null controllable if (5) (i) is violated (see Remark 3.9). On the other hand, for specific problems like the Schrödinger equation 
one can allow for (5) (i) to be violated for a finite number of eigenstates assuming additional conditions (see [6]).

The above stabilizability result can be used to study several classes of parabolic problems, for which checking the validity of the assumptions on $A$ and $B$ is usually straightforward. For instance, we can treat the heat equation with a controlled source term of the form

$$
u_{t}-u_{x x}+p(t) \mu(x) u=0
$$

with Dirichlet or Neumann boundary conditions, as well as operators with variable coefficients

$$
u_{t}-\left((1+x)^{2} u_{x}\right)_{x}+p(t) \mu(x) u=0,
$$

or even $3 D$ problems with radial data symmetry such as

$$
u_{t}-\Delta u+p(t) \mu(|x|) u=0 .
$$

The above examples are restricted to one space dimension because of the gap condition in Theorem 3.10. On the other hand, the control we construct is just a scalar function of time. As such, its influence on the system is necessarily limited. In order to treat problems in higher dimension, one should probably use bilinear controls depending on both time and space (see, e.g., $[9,17])$.

The results of this paper have been applied to degenerate parabolic equations in [12]. Moreover, our method can be adapted to obtain exact controllability to eigensolutions (see [1]) under slightly more restrictive assumptions, as well as to treat equations with an unbounded coefficient $B$ such as the Fokker-Planck equations (see [2]).

The outline of the paper is the following. In Sect. 2, we introduce the notation and the preliminary assumptions on the data. Section 3 is devoted to our main results. In Sects. 4 and 5, we show the proofs of Theorems 3.7 and 3.8. Finally, in Sect. 6 we give applications to several examples of parabolic problems.

\section{Preliminaries}

Let $(X,\langle\cdot, \cdot\rangle)$ be a separable Hilbert space. We denote by $\|\cdot\|$ the associated norm on $X$ and by $B_{R}(\varphi)$ the open ball of radius $R>0$, centered in $\varphi \in X$.

Let $A: D(A) \subset X \rightarrow X$ be a densely defined, linear operator with the following properties:

(a) $A$ is self-adjoint,

(b) $\exists \sigma>0:\langle A x, x\rangle \geq-\sigma\|x\|^{2}, \forall x \in D(A)$,

(c) $\exists \lambda>-\sigma$ such that $(\lambda I+A)^{-1}: X \rightarrow X$ is compact.

We recall that under the above assumptions $A$ is a closed operator and $D(A)$ is itself a Hilbert space with the scalar product

$$
(x \mid y)_{D(A)}=\langle x, y\rangle+\langle A x, A y\rangle, \quad \forall x, y \in D(A) .
$$


Moreover, $-A$ is the infinitesimal generator of a strongly continuous semigroup on $X$ which will be denoted by $\mathrm{e}^{-t A}$.

Let $\mathbb{N}^{*}$ denote the real numbers greater or equal than 1 . In view of the above assumptions, there exists an orthonormal basis $\left\{\varphi_{k}\right\}_{k \in \mathbb{N}^{*}}$ in $X$ of eigenfunctions of $A$, that is, $\varphi_{k} \in D(A),\left\|\varphi_{k}\right\|=1$ and $A \varphi_{k}=\lambda_{k} \varphi_{k}, \forall k \in \mathbb{N}^{*}$, where $\left\{\lambda_{k}\right\}_{k \in \mathbb{N}^{*}} \subset \mathbb{R}$ denote the corresponding eigenvalues. We recall that $\lambda_{k} \geq-\sigma, \forall k \in \mathbb{N}^{*}$, and we supposewithout loss of generality-that $\left\{\lambda_{k}\right\}_{k \in \mathbb{N}^{*}}$ is ordered so that $-\sigma \leq \lambda_{k} \leq \lambda_{k+1} \rightarrow \infty$ as $k \rightarrow \infty$.

The associated semigroup has the following representation:

$$
\mathrm{e}^{-t A} \varphi=\sum_{k=1}^{\infty}\left\langle\varphi, \varphi_{k}\right\rangle \mathrm{e}^{-\lambda_{k} t} \varphi_{k}, \quad \forall \varphi \in X .
$$

Let $T>0$ and consider the problem

$$
\left\{\begin{array}{l}
u^{\prime}(t)+A u(t)=f(t), \quad t \in[0, T] \\
u(0)=u_{0}
\end{array}\right.
$$

where $u_{0} \in X$ and $f \in L^{2}(0, T ; X)$. We now recall two definitions of solution of problem (8) (see, e.g., [7, p. 129]):

- the function $u \in C([0, T], X)$ defined by

$$
u(t)=\mathrm{e}^{-t A} u_{0}+\int_{0}^{t} \mathrm{e}^{-(t-s) A} f(s) \mathrm{d} s
$$

is called the mild solution of (8),

- $u$ is a strong solution of $(8)$ in $L^{2}(0, T ; X)$ if there exists a sequence $\left\{u_{k}\right\} \subseteq$ $H^{1}(0, T ; X) \cap L^{2}(0, T ; D(A))$ such that

$$
\begin{gathered}
u_{k} \rightarrow u, \text { and } u_{k}^{\prime}-A u_{k} \rightarrow f \text { in } L^{2}(0, T ; X), \\
u_{k}(0) \rightarrow u_{0} \text { in } X, \text { as } k \rightarrow \infty .
\end{gathered}
$$

The well-posedness of the Cauchy problem (8) is a classical result (see, for instance, [7, Proposition 3.1, p. 130]).

Theorem 2.1. Let $u_{0} \in X$ and $f \in L^{2}(0, T ; X)$. Under hypothesis (6), problem (8) has a unique strong solution in $L^{2}(0, T ; X)$. Moreover, $u$ belongs to $C([0, T] ; X)$ and coincides with the mild solution (9).

Furthermore, there exists a constant $C_{0}(T)>0$ such that

$$
\sup _{t \in[0, T]}\|u(t)\| \leq C_{0}(T)\left(\left\|u_{0}\right\|+\|f\|_{L^{2}(0, T ; X)}\right)
$$

and $C_{0}(T)$ is nondecreasing with respect to $T$. 


\section{Main result}

We consider the bilinear control problem

$$
\left\{\begin{array}{l}
u^{\prime}(t)+A u(t)+p(t) B u(t)=0, \quad t>0 \\
u(0)=u_{0}
\end{array}\right.
$$

where $u$ is the state variable and $p \in L_{\mathrm{loc}}^{2}([0,+\infty))$ is the control function.

We recall that, in general, the exact controllability problem for system (11) has a negative answer when $B$ is a bounded linear operator, as shown by Ball, Marsden and Slemrod in [3].

We are interested in studying the stabilizability of system (11) to a fixed trajectory. Given an initial condition $u_{0} \in X$ and a control $p \in L_{\text {loc }}^{2}([0,+\infty))$, we denote by $u\left(\cdot ; u_{0}, p\right):[0,+\infty) \rightarrow X$ the corresponding solution of (11).

Definition 3.1. Given $\bar{u}_{0} \in X$ and $\bar{p} \in L_{\text {loc }}^{2}([0,+\infty))$, we say that the control system (11) is locally stabilizable to $\bar{u}\left(\cdot ; \bar{u}_{0}, \bar{p}\right)$ if there exists $\delta>0$ such that, for every $u_{0} \in B_{\delta}\left(\bar{u}_{0}\right)$, there exists a control $p \in L_{\text {loc }}^{2}([0,+\infty))$ such that

$$
\lim _{t \rightarrow+\infty}\left\|u\left(t ; u_{0}, p\right)-\bar{u}\left(t ; \bar{u}_{0}, \bar{p}\right)\right\|=0 .
$$

Definition 3.2. Given $\bar{u}_{0} \in X$ and $\bar{p} \in L_{\text {loc }}^{2}([0,+\infty))$, we say that the control system (11) is locally exponentially stabilizable to $\bar{u}\left(\cdot ; \bar{u}_{0}, \bar{p}\right)$ if for any $\rho>0$, there exists $R(\rho)>0$ such that, for every $u_{0} \in B_{R(\rho)}\left(\bar{u}_{0}\right)$, there exists a control $p \in L_{\text {loc }}^{2}([0,+\infty))$ such that

$$
\left\|u\left(t ; u_{0}, p\right)-\bar{u}\left(t ; \bar{u}_{0}, \bar{p}\right)\right\| \leq M \mathrm{e}^{-\rho t}, \quad \forall t>0
$$

where $M>0$ is a constant that depends only on $A$ and $B$.

Definition 3.3. Given $\bar{u}_{0} \in X$ and $\bar{p} \in L_{\text {loc }}^{2}([0,+\infty))$, we say that the control system (11) is locally superexponentially stabilizable to $\bar{u}\left(\cdot ; \bar{u}_{0}, \bar{p}\right)$ if for any $\rho>0$ there exists $R(\rho)>0$ such that, for every $u_{0} \in B_{R(\rho)}\left(\bar{u}_{0}\right)$, there exists a control $p \in$ $L_{\text {loc }}^{2}([0,+\infty))$ such that

$$
\left\|u\left(t ; u_{0}, p\right)-\bar{u}\left(t ; \bar{u}_{0}, \bar{p}\right)\right\| \leq M \mathrm{e}^{-\rho \mathrm{e}^{\omega t}}, \quad \forall t>0
$$

where $M, \omega>0$ are suitable constants depending only on $A$ and $B$.

For any $j \in \mathbb{N}^{*}$, we recall that we set $\psi_{j}(t)=\mathrm{e}^{-\lambda_{j} t} \varphi_{j}$ and we call $\psi_{j}$ the $j$ th eigensolution of $A$. Observe that $\psi_{j}$ solves (11) with $p=0$ and $u_{0}=\varphi_{j}$. We shall study the superexponential stabilizability of (11) to $\psi_{j}$.

We observe that, at least for strictly accretive operators $A$, system (11) is trivially locally exponentially stabilizable to any trajectory $\psi_{j}$ by choosing the control $p=0$.

The novelty of our work is the construction of a control function $p$ that steers $u(t)$ arbitrary close to $\psi_{j}(t)$ at superexponential speed of decay. 
Let $B: X \rightarrow X$ be a bounded linear operator. From now on, we denote by $C_{B}$ the norm of $B$, that is,

$$
C_{B}=\sup _{\varphi \in X,\|\varphi\|=1}\|B \varphi\|
$$

and, without loss of generality, we suppose $C_{B} \geq 1$.

Let $A: D(A) \subset X \rightarrow X$ be a densely defined linear operator such that (6) hold.

Definition 3.4. Let $T>0$. The pair $\{A, B\}$ is called $j$-null controllable in time $T$ if there exists a constant $N(T)>0$ such that for every $y_{0} \in X$ one can find a control $p \in L^{2}(0, T)$ satisfying

$$
\|p\|_{L^{2}(0, T)} \leq N(T)\left\|y_{0}\right\|
$$

and for which $y(T)=0$, where $y(\cdot)$ is the solution of

$$
\left\{\begin{array}{l}
y^{\prime}(t)+A y(t)+p(t) B \varphi_{j}=0, \quad t \in[0, T] \\
y(0)=y_{0} .
\end{array}\right.
$$

$N(T)>0$ is called the control cost.

Definition 3.5. The pair $\{A, B\}$ is called $j$-null controllable if there exists $T_{0}>0$ such that $\{A, B\}$ is $j$-null controllable in time $T_{0}$.

Remark 3.6. Observe that if $\{A, B\}$ is $j$-null controllable in time $T_{0}$, it is $j$-null controllable in any time $T>T_{0}$.

We can now state our main result.

Theorem 3.7. Let $\{A, B\}$ be a $j$-null controllable pair. Then, system (11) is superexponentially stabilizable to $\psi_{j}$, for any $j \geq 1$.

In the following result, we give sufficient conditions for $j$-null controllability.

Theorem 3.8. Let $A: D(A) \subset X \rightarrow X$ be a densely defined linear operator that satisfies (6) and such that there exists a constant $\alpha>0$ for which its eigenvalues fulfill the gap condition

$$
\sqrt{\lambda_{k+1}-\lambda_{1}}-\sqrt{\lambda_{k}-\lambda_{1}} \geq \alpha, \quad \forall k \in \mathbb{N}^{*} .
$$

Let $B: X \rightarrow X$ be a bounded linear operator such that

$$
\begin{aligned}
& \text { (i) }\left\langle B \varphi_{j}, \varphi_{k}\right\rangle \neq 0, \quad \forall k \in \mathbb{N}^{*}, \\
& \text { (ii) } \exists \tau>0: \sum_{k \in \mathbb{N}^{*}} \frac{\mathrm{e}^{-2 \lambda_{k} \tau}}{\left|\left\langle B \varphi_{j}, \varphi_{k}\right\rangle\right|^{2}}<+\infty .
\end{aligned}
$$

Then, the pair $\{A, B\}$ is $j$-null controllable. 
Remark 3.9. We observe that assumption (16) (i) is necessary for the null controllability of the linear system (14). Indeed, fixed any $T>0$ and $y_{0} \in X$, suppose that there exists $p \in L^{2}(0, T)$ such that $y(T)=0$. Taking the Fourier expansion of the solution of (14), thanks to (7) and (9), the previous identity is equivalent to

$$
\sum_{k \in \mathbb{N}^{*}}\left\langle y_{0}, \varphi_{k}\right\rangle \mathrm{e}^{-\lambda_{k} T} \varphi_{k}=\int_{0}^{T} p(s) \sum_{k \in \mathbb{N}^{*}}\left\langle B \varphi_{j}, \varphi_{k}\right\rangle \mathrm{e}^{-\lambda_{k}(T-s)} \varphi_{k} \mathrm{~d} s .
$$

Since the family $\left\{\varphi_{k}\right\}_{k \in \mathbb{N}^{*}}$ forms an othonormal basis of $X$, it holds that

$$
\left\langle y_{0}, \varphi_{k}\right\rangle=\int_{0}^{T} \mathrm{e}^{\lambda_{k} s} p(s)\left\langle B \varphi_{j}, \varphi_{k}\right\rangle \mathrm{d} s, \quad \forall k \in \mathbb{N}^{*} .
$$

In particular, if (16) (i) is violated, there exists some $\bar{k} \in \mathbb{N}^{*}$ such that $\left\langle B \varphi_{j}, \varphi_{\bar{k}}\right\rangle=0$. Hence, in the $\bar{k}$ th direction we have that

$$
\left\langle y_{0}, \varphi_{\bar{k}}\right\rangle=\int_{0}^{T} \mathrm{e}^{\lambda_{\bar{k}} s} p(s)\left\langle B \varphi_{j}, \varphi_{\bar{k}}\right\rangle \mathrm{d} s,
$$

which yields to $y_{0} \in \varphi_{\bar{k}}^{\perp}$.

From Theorems 3.7 and 3.8 , we easily deduce the following result:

Corollary 3.10. Let $A: D(A) \subset X \rightarrow X$ be a densely defined linear operator that satisfies (6) and (15). Let $B: X \rightarrow X$ be a bounded linear operator such that (16) holds.

Then, problem (11) is locally superexponentially stabilizable to any eigensolution $\psi_{j}, j \geq 1$.

\section{Proof of Theorem 3.7}

The proof of Theorem 3.7 will be built through a series of propositions. The first result is the well-posedness of the problem

$$
\left\{\begin{array}{l}
u^{\prime}(t)+A u(t)+p(t) B u(t)+f(t)=0, \quad t \in[0, T] \\
u(0)=u_{0}
\end{array}\right.
$$

We introduce the following notation:

$$
\begin{aligned}
& \|f\|_{2,0}:=\|f\|_{L^{2}(0, T ; X)}, \quad \forall f \in L^{2}(0, T ; X), \\
& \|f\|_{\infty, 0}:=\|f\|_{C([0, T] ; X)}=\sup _{t \in[0, T]}\|f(t)\|, \quad \forall f \in C([0, T] ; X) .
\end{aligned}
$$




\subsection{Well-posedness}

Proposition 4.1. Let $T>0$. If $u_{0} \in X, p \in L^{2}(0, T)$ and $f \in L^{2}(0, T ; X)$, then there exists a unique mild solution of (17), i.e., a function $u \in C([0, T] ; X)$ such that

$$
u(t)=\mathrm{e}^{-t A} u_{0}-\int_{0}^{t} \mathrm{e}^{-(t-s) A}[p(s) B u(s)+f(s)] \mathrm{d} s, \quad \forall t \in[0, T] .
$$

Moreover, there exists a constant $C_{1}(T)>0$ such that

$$
\|u\|_{\infty, 0} \leq C_{1}(T)\left(\left\|u_{0}\right\|+\|f\|_{2,0}\right) .
$$

Hereafter, we denote by $C$ a generic positive constant which may differ from line to line even if the symbol remains the same. Constants which play a specific role will be distinguished by an index, i.e., $C_{0}, C_{B}, \ldots$

The proof of the existence of the mild solution of (17) is given in [3]. For what concerns the bound for the solution $u$ of (17), it turns out that, by applying estimate (10) of Theorem 2.1 to (17), if $C_{0}(T) C_{B}\|p\|_{L^{2}(0, T)} \leq 1 / 2$, then we have inequality (19) with $C_{1}=C_{2}$ defined by

$$
C_{2}:=2 C_{0}(T)
$$

where $C_{0}(T)$ is introduced in (10). Otherwise, to obtain (19), we proceed by subdividing the interval $[0, T]$ into smaller subintervals for which $C_{0}(T) C_{B}\|p\|_{L^{2}} \leq 1 / 2$ in all of them. In this case, the constant $C_{1}$ in inequality (19) is defined by

$$
C_{1}=(1+m)\left(2 C_{0}(T / m)\right)^{m},
$$

where $m$ is the number of subintervals.

To prove Theorem 3.7, we first start by assuming $\{A, B\}$ to be $j$-null controllable and such that the $j$ th eigenvalue of $A$ is zero, that is $\lambda_{j}=0$, and we prove the local superexponential stabilizability of (11) to the trajectory $\varphi_{j}$. Then, we will recover the case $\lambda_{j} \neq 0$ and the stabilization to any trajectory $\psi_{j}$ from the previous one.

4.2. Uniform estimate for the solution of the nonlinear system

Consider the system

$$
\left\{\begin{array}{l}
u^{\prime}(t)+A u(t)+p(t) B u(t)=0, t \in[0, T] \\
u(0)=u_{0}
\end{array}\right.
$$

and the trajectory $\varphi_{j}$ that is a solution of (22) when $p=0, u_{0}=\varphi_{j}$ and $\lambda_{j}=0$. Setting $v:=u-\varphi_{j}$, we observe that $v$ is the solution of the following Cauchy problem:

$$
\left\{\begin{array}{l}
v^{\prime}(t)+A v(t)+p(t) B v(t)+p(t) B \varphi_{j}=0, \quad t \in[0, T] \\
v(0)=v_{0}:=u_{0}-\varphi_{j}
\end{array}\right.
$$


Remark 4.2. Applying Theorem 2.1, we find that $v \in C([0, T] ; X)$ is a mild solution of (23), that is

$$
v(t)=\mathrm{e}^{-t A} v_{0}-\int_{0}^{t} p(s) \mathrm{e}^{-(t-s) A} B\left(v(s)+\varphi_{j}\right) \mathrm{d} s=V_{0}(t)+V_{1}(t),
$$

where

$$
\begin{aligned}
& V_{0}(t):=\mathrm{e}^{-t A} v_{0}, \\
& V_{1}(t):=-\int_{0}^{t} p(s) \mathrm{e}^{-(t-s) A} B\left(v(s)+\varphi_{j}\right) \mathrm{d} s .
\end{aligned}
$$

Since $p(\cdot) B\left(v(\cdot)+\varphi_{j}\right) \in L^{2}(0, T ; X)$, recalling Theorem 2.1 , we have that $V_{1} \in$ $H^{1}(0, T ; X) \cap L^{2}(0, T ; D(A))$, while $V_{0} \in C^{1}((0, T] ; X) \cap C((0, T] ; D(A))$. Therefore, for every $\varepsilon \in(0, T), v \in H^{1}(\varepsilon, T ; X) \cap L^{2}(\varepsilon, T ; D(A))$ and for almost every $t \in[\varepsilon, T]$ the following equality holds:

$$
v^{\prime}(t)+A v(t)+p(t) B v(t)+p(t) B \varphi_{j}=0
$$

Showing the stabilizability of the solution $u$ of (22) to the trajectory $\varphi_{j}$ is equivalent to proving the stabilizability to 0 of system (23).

Proposition 4.3. Let $\{A, B\}$ be $j$-null controllable in time $T_{0}$ and assume $\lambda_{j}=0$. Let $p_{0} \in L^{2}\left(0, T_{0}\right)$ be a control satisfying (13) such that $\bar{v}\left(T_{0}\right)=0$, where $\bar{v}(\cdot)$ is the solution of the following linear system:

$$
\left\{\begin{array}{l}
\bar{v}(t)^{\prime}+A \bar{v}(t)+p_{0}(t) B \varphi_{j}=0, \quad t \in\left[0, T_{0}\right] \\
\bar{v}(0)=v_{0}
\end{array}\right.
$$

Then, the solution $v$ of (23) with $p=p_{0}$ and $T=T_{0}$ satisfies

$$
\sup _{t \in\left[0, T_{0}\right]}\|v(t)\|^{2} \leq \mathrm{e}^{\left(2 \sigma+C_{B}\right) T_{0}+2 C_{B} N\left(T_{0}\right) \sqrt{T_{0}}\left\|v_{0}\right\|}\left(1+C_{B} N^{2}\left(T_{0}\right)\right)\left\|v_{0}\right\|^{2},
$$

where $C_{B}=\|B\| \geq 1, \sigma$ is defined in (6) and $N\left(T_{0}\right)$ is the control cost in (13).

Proof. Thanks to Remark 4.2, taking the scalar product of (25) with $v$, we obtain

$$
\left\langle v^{\prime}(t), v(t)\right\rangle+\langle A v(t), v(t)\rangle+p_{0}(t)\left\langle B v(t)+B \varphi_{j}, v(t)\right\rangle=0, \quad \text { for a.e. } t \in\left[\varepsilon, T_{0}\right]
$$

Thus, we get that for a.e. $t \in\left[\varepsilon, T_{0}\right]$

$$
\begin{aligned}
\frac{1}{2} \frac{d}{d t}\|v(t)\|^{2}+\langle A v(t), v(t)\rangle & \leq C_{B}\left(\left|p_{0}(t)\right|\|v(t)\|^{2}+\left|p_{0}(t)\right|\left\|\varphi_{j}\right\|\|v(t)\|\right) \\
& \leq C_{B}\left(\left|p_{0}(t)\right|\|v(t)\|^{2}+\frac{1}{2}\left|p_{0}(t)\right|^{2}+\frac{1}{2}\|v(t)\|^{2}\right) .
\end{aligned}
$$


Therefore, since $A$ satisfies (6), we have that

$$
\frac{1}{2} \frac{d}{d t}\|v(t)\|^{2} \leq\left(\sigma+C_{B}\left|p_{0}(t)\right|+\frac{C_{B}}{2}\right)\|v(t)\|^{2}+\frac{1}{2} C_{B}\left|p_{0}(t)\right|^{2}, \quad \text { for a.e. } t \in\left[\varepsilon, T_{0}\right] .
$$

We now integrate the last inequality from $\varepsilon$ to $t$ to obtain

$$
\begin{aligned}
& \int_{\varepsilon}^{t} \frac{d}{d s}\|v(s)\|^{2} \mathrm{~d} s \\
& \quad \leq \int_{\varepsilon}^{t}\left(2 \sigma+C_{B}\left(2\left|p_{0}(s)\right|+1\right)\right)\|v(s)\|^{2} \mathrm{~d} s+C_{B} \int_{0}^{T_{0}}\left|p_{0}(s)\right|^{2} \mathrm{~d} s, \quad \text { for a.e. } t \in\left[\varepsilon, T_{0}\right],
\end{aligned}
$$

and, by Gronwall's inequality, we conclude that

$\|v(t)\|^{2} \leq\left(\|v(\varepsilon)\|^{2}+C_{B} \int_{0}^{T_{0}}\left|p_{0}(s)\right|^{2} \mathrm{~d} s\right) \mathrm{e}^{\int_{\varepsilon}^{t}\left(2 \sigma+C_{B}\left(2\left|p_{0}(s)\right|+1\right)\right)} \mathrm{d} s, \quad$ for a.e. $t \in\left[\varepsilon, T_{0}\right]$.

Taking the limit as $\varepsilon \rightarrow 0$, we find

$$
\|v(t)\|^{2} \leq\left(\left\|v_{0}\right\|^{2}+C_{B} \int_{0}^{T_{0}}\left|p_{0}(s)\right|^{2} \mathrm{~d} s\right) \mathrm{e}^{\int_{0}^{t}\left(2 \sigma+C_{B}\left(2\left|p_{0}(s)\right|+1\right)\right)} \mathrm{d} s, \quad \text { for a.e. } t \in\left[0, T_{0}\right] .
$$

Thus, taking the supremum over the interval $\left[0, T_{0}\right]$, the last inequality becomes

$$
\sup _{t \in\left[0, T_{0}\right]}\|v(t)\|^{2} \leq \mathrm{e}^{2 C_{B} \sqrt{T_{0}}\left\|p_{0}\right\|_{L^{2}\left(0, T_{0}\right)}+\left(2 \sigma+C_{B}\right) T_{0}}\left(\left\|v_{0}\right\|^{2}+C_{B}\left\|p_{0}\right\|_{L^{2}\left(0, T_{0}\right)}^{2}\right) .
$$

Finally, recalling estimate (13) for $p_{0}$, we get (27).

4.3. Quadratic estimate for the solution of the nonlinear system

Let $p_{0} \in L^{2}\left(0, T_{0}\right)$ be as in Proposition 4.3. We introduce the function $w(t):=$ $v(t)-\bar{v}(t)$, where $v$ is the solution of (23) with $p=p_{0}$ and $T=T_{0}$ and $\bar{v}$ is the solution of (26). Then, $w$ satisfies the following Cauchy problem:

$$
\left\{\begin{array}{l}
w^{\prime}(t)+A w(t)+p_{0}(t) B v(t)=0, \quad t \in\left[0, T_{0}\right] \\
w(0)=0
\end{array}\right.
$$

We define the constant

$$
K_{0}^{2}:=C_{B}^{2} N^{2}\left(T_{0}\right) \mathrm{e}^{2\left(2 \sigma+C_{B}\right) T_{0}+2 C_{B} \sqrt{T_{0}}}\left(1+C_{B} N^{2}\left(T_{0}\right)\right),
$$

where we recall that $\sigma, C_{B}$ and $N\left(T_{0}\right)$ are defined in (6), (12) and (13) respectively.

Proposition 4.4. Let $\{A, B\}$ be $j$-null controllable in time $T_{0}$ and assume $\lambda_{j}=0$. If

$$
K_{0}\left\|v_{0}\right\| \leq 1
$$

then the solution of (30) satisfies

$$
\left\|w\left(T_{0}\right)\right\| \leq K_{0}\left\|v_{0}\right\|^{2} .
$$


Proof. Since $w \in H^{1}\left(0, T_{0} ; X\right) \cap L^{2}\left(0, T_{0} ; D(A)\right)$, taking the scalar product of both members of the equation in (30) with $w(t)$, we obtain

$$
\begin{aligned}
\frac{1}{2} \frac{d}{d t}\|w(t)\|^{2} & \leq \sigma\|w(t)\|^{2}+\left|p_{0}(t)\||| B v(t)\|\|w(t)\|\right. \\
& \leq\left(\frac{1}{2}+\sigma\right)\|w(t)\|^{2}+C_{B}^{2} \frac{1}{2}\left|p_{0}(t)\right|^{2}\|v(t)\|^{2}, \quad \text { for a.e } t \in\left[0, T_{0}\right]
\end{aligned}
$$

Then, by Gronwall's inequality, and appealing to (27) and (13), we get

$$
\begin{aligned}
\sup _{t \in\left[0, T_{0}\right]}\|w(t)\|^{2} & \leq C_{B}^{2} \mathrm{e}^{(2 \sigma+1) T_{0}}\left\|p_{0}\right\|_{L^{2}\left(0, T_{0}\right)}^{2} \sup _{t \in\left[0, T_{0}\right]}\|v(t)\|^{2} \\
& \leq C_{B}^{2} \mathrm{e}^{\left(4 \sigma+C_{B}+1\right) T_{0}+2 C_{B} N\left(T_{0}\right) \sqrt{T_{0}}\left\|v_{0}\right\|}\left(1+C_{B} N^{2}\left(T_{0}\right)\right)\left\|v_{0}\right\|^{2}\left\|p_{0}\right\|_{L^{2}\left(0, T_{0}\right)}^{2} \\
& \leq C_{B}^{2} N^{2}\left(T_{0}\right) \mathrm{e}^{\left(4 \sigma+C_{B}+1\right) T_{0}+2 C_{B} N\left(T_{0}\right) \sqrt{T_{0}}\left\|v_{0}\right\|}\left(1+C_{B} N^{2}\left(T_{0}\right)\right)\left\|v_{0}\right\|^{4} .
\end{aligned}
$$

Thus, from (32), we obtain that $N\left(T_{0}\right)\left\|v_{0}\right\| \leq 1$. Therefore,

$$
\sup _{t \in\left[0, T_{0}\right]}\|w(t)\|^{2} \leq K_{0}^{2}\left\|v_{0}\right\|^{4}
$$

which yields to (33).

4.4. Iterated quadratic estimate and Proof of Theorem 3.7

Recalling that $\bar{v}\left(T_{0}\right)=0$, we deduce from (33) that

$$
\left\|v\left(T_{0}\right)\right\| \leq K_{0}\left\|v_{0}\right\|^{2} .
$$

Now, we want to obtain an estimate as (36) for the solution $v$ of problem (23) defined in successive time intervals of the form $[n T,(n+1) T]$, with $n \geq 1$.

Proposition 4.5. Let $\{A, B\}$ be $j$-null controllable in time $T_{0}$ and assume $\lambda_{j}=0$. Let $v_{0} \in X$ be such that

$$
K_{0}\left\|v_{0}\right\| \leq 1
$$

where $K_{0}$ is defined in (31).

Then, there exists a control $p_{n} \in L^{2}\left(n T_{0},(n+1) T_{0}\right)$ such that the corresponding solution $v$ of $(23)$ on the time interval $\left[n T_{0},(n+1) T_{0}\right]$ satisfies

$$
\left\|v\left((n+1) T_{0}\right)\right\| \leq \frac{1}{K_{0}}\left(K_{0}\left\|v_{0}\right\|\right)^{2^{n+1}}, \quad \forall n \geq 0 .
$$

Proof. We proceed by induction on $n$. For $n=0$, the result has been proved in Proposition 4.4. We suppose that it is true for any index until $n-1$, and we prove it for $n$. Iterating the construction of the solution $v$ of (23) in consecutive time intervals 
of the form $\left[k T_{0},(k+1) T_{0}\right]$ until $k+1=n$, we set $v_{n T_{0}}=v\left(n T_{0}\right)$ and we consider the system

$$
\left\{\begin{array}{l}
v^{\prime}(t)+A v(t)+p_{n}(t) B v(t)+p_{n}(t) B \varphi_{j}=0, \quad t \in\left[n T_{0},(n+1) T_{0}\right], \\
v\left(n T_{0}\right)=v_{n T_{0}} .
\end{array}\right.
$$

We shift this problem in the time interval $\left[0, T_{0}\right]$ by introducing the variable $s:=$ $t-n T_{0}$ and the functions $\tilde{v}(s)=v\left(s+n T_{0}\right), \tilde{p}_{n}(s)=p_{n}\left(s+n T_{0}\right)$. Then, $\tilde{v}$ is the solution of the following Cauchy problem:

$$
\left\{\begin{array}{l}
\tilde{v}_{t}(s)+A \tilde{v}(s)+\tilde{p}_{n}(s) B \tilde{v}(s)+\tilde{p}_{n}(s) B \varphi_{j}=0, \quad s \in\left[0, T_{0}\right] \\
\tilde{v}(0)=v_{n T_{0}}
\end{array}\right.
$$

Since $\{A, B\}$ is $j$-null controllable in time $T_{0}$, there exists a control $\tilde{p}_{n} \in L^{2}\left(0, T_{0}\right)$ such that the associated linear system of (40) is null controllable and moreover $\tilde{p}_{n}$ satisfies

$$
\left\|\tilde{p_{n}}\right\|_{L^{2}(0, T)} \leq N\left(T_{0}\right)\left\|v_{n T_{0}}\right\| .
$$

Therefore, coming back to the original time interval $\left[n T_{0},(n+1) T_{0}\right]$ and setting $p_{n}(t):=\tilde{p}_{n}\left(t-n T_{0}\right)$, for all $t \in\left[n T_{0},(n+1) T_{0}\right]$, we find that

$$
\left\|p_{n}\right\|_{L^{2}\left(n T_{0},(n+1) T_{0}\right)}=\left\|\tilde{p}_{n}\right\|_{L^{2}\left(0, T_{0}\right)} \leq N\left(T_{0}\right)\left\|v_{n T_{0}}\right\| .
$$

Moreover, since by inductive hypothesis

$$
\left\|v\left(n T_{0}\right)\right\| \leq \frac{1}{K_{0}}\left(K_{0}\left\|v_{0}\right\|\right)^{2^{n}}
$$

we deduce that

$$
K_{0}\left\|v\left(n T_{0}\right)\right\| \leq 1
$$

Hence, we can use Proposition 4.4 for problem (39), obtaining

$$
\left\|v\left((n+1) T_{0}\right)\right\| \leq K_{0}\left\|v\left(n T_{0}\right)\right\|^{2} \leq K_{0}\left(\frac{1}{K_{0}}\left(K_{0}\left\|v_{0}\right\|\right)^{2^{n}}\right)^{2}=\frac{1}{K_{0}}\left(K_{0}\left\|v_{0}\right\|\right)^{2^{n+1}(44)}
$$

and this concludes the induction argument and the proof of the proposition.

The last step that allows us to prove Theorem 3.7 consists in showing the rapid decay of the solution $u$ of our initial problem (11) to the fixed stationary trajectory $\varphi_{j}$.

Proposition 4.6. Let $\{A, B\}$ be $j$-null controllable in time $T_{0}$ and assume $\lambda_{j}=0$.

Then, there exists a constant $C_{T_{0}}>0$ such that for every $\theta \in(0,1)$ and for every $\left\|v_{0}\right\| \leq \frac{\theta}{K_{0}}$, with $K_{0}$ defined as in (31), there exists a control $p \in L_{\mathrm{loc}}^{2}(0,+\infty)$ for which the solution $u$ of (11) satisfies

$$
\left\|u(t)-\varphi_{j}\right\| \leq \frac{C_{T_{0}}}{K_{0}} \theta^{2^{\frac{t}{T_{0}}-1}}, \quad \forall t \geq 0 .
$$


Proof. We have supposed that $\left\|v_{0}\right\| \leq \frac{\theta}{K_{0}}$, with $\theta \in(0,1)$. Thus, (38) at rank $n-1$, becomes

$$
\left\|v\left(n T_{0}\right)\right\| \leq \frac{\theta^{2^{n}}}{K_{0}}, \quad \forall n \geq 1 .
$$

Consider now the time interval $\left[n T_{0},(n+1) T_{0}\right]$. From estimate (19) used here for problem (39) in the time interval $\left[n T_{0},(n+1) T_{0}\right]$ and from the bound (41) for the control $p_{n}$, we deduce that there exists a constant $C_{T_{0}}>0$ such that

$$
\|v(t)\| \leq C_{T_{0}}\left\|v\left(n T_{0}\right)\right\|, \quad \forall t \in\left[n T_{0},(n+1) T_{0}\right] .
$$

Therefore, using (38) in (47), we obtain that

$$
\|v(t)\| \leq C_{T_{0}}\left\|v\left(n T_{0}\right)\right\| \leq \frac{C_{T_{0}}}{K_{0}} \theta^{2^{n}}=\frac{C_{T_{0}}}{K_{0}}\left(\theta^{2^{(n+1)}}\right)^{1 / 2}, \quad \forall t \in\left[n T_{0},(n+1) T_{0}\right] .
$$

Since $n \leq \frac{t}{T_{0}} \leq(n+1)$ and $\theta \in(0,1)$, it holds that

$$
\|v(t)\| \leq \frac{C_{T_{0}}}{K_{0}}\left(\theta^{2^{(n+1)}}\right)^{1 / 2} \leq \frac{C_{T_{0}}}{K_{0}}\left(\theta^{2^{\frac{t}{T_{0}}}}\right)^{1 / 2}=\frac{C_{T_{0}}}{K_{0}} \theta^{2^{\frac{t}{T_{0}}-1}}, \quad \forall t \geq 0 .
$$

By definition, $v(t)=u(t)-\varphi_{j}$. So, we deduce (45).

We now prove Theorem 3.7 for $\lambda_{j}=0$.

Proof of Theorem 3.7 (case $\lambda_{j}=0$ ) Since $\{A, B\}$ is by assumption $j$-null controllable, there exists $T_{0}>0$ such that $\{A, B\}$ is $j$-null controllable in time $T_{0}$. By hypothesis, the $j$ th eigenvalue of $A$ is equal to zero; hence, we have to prove the superexponential stabilizability of system (22) to the eigensolution $\psi_{j} \equiv \varphi_{j}$.

Let $\rho>0$ and let $\theta \in(0,1)$ be the value for which $\theta=\mathrm{e}^{-2 \rho}$. Then, setting $R_{\rho}:=\frac{\mathrm{e}^{-2 \rho}}{K_{0}}$, from Proposition 4.6, if $\left\|u_{0}-\varphi_{j}\right\| \leq R_{\rho}$, then

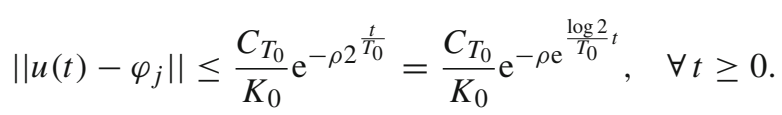

Hence, by defining

$$
M:=\frac{C_{T_{0}}}{K_{0}}, \quad \omega:=\frac{\log 2}{T_{0}},
$$

we conclude the proof of local superexponential stabilizability of problem (11) to the $j$ th eigensolution, when $\lambda_{j}=0$, by means of a control $p \in L_{\text {loc }}^{2}(0,+\infty)$ defined as

$$
p(t)=\sum_{n=0}^{\infty} p_{n}(t) \chi_{\left[n T_{0},(n+1) T_{0}\right]}(t) .
$$

We now deal with the general case $\lambda_{j} \neq 0$. 
Lemma 4.7. Let A satisfy hypothesis (6). If $\{A, B\}$ is $j$-null controllable, then $\left\{A_{j}, B\right\}$ is $j$-null controllable, where $A_{j}: D(A) \subset X \rightarrow X$ is defined as

$$
A_{j}:=A-\lambda_{j} I
$$

Proof. It is immediate to check that $A_{j}$ verifies (6). Let us show that $\left\{A_{j}, B\right\}$ is $j$-null controllable in the same time $T_{0}>0$ as $\{A, B\}$.

We have to prove that the following problem

$$
\left\{\begin{array}{l}
y^{\prime}(t)+A_{j} y(t)+q(t) B \varphi_{j}=0, \quad t \in[0, T], \\
y(0)=y_{0} \in X
\end{array}\right.
$$

is null controllable and that the desired estimate (13) on $q$ holds true. Now, defining $Y(t):=\mathrm{e}^{-\lambda_{j} t} y(t)$ and $p(t):=\mathrm{e}^{-\lambda_{j} t} q(t)$, we can rewrite problem (52) as

$$
\left\{\begin{array}{l}
Y^{\prime}(t)+A Y(t)+p(t) B \varphi_{j}=0, \quad t \in[0, T] \\
Y(0)=y_{0}
\end{array}\right.
$$

Since $\{A, B\}$ is $j$-null controllable, there exists a time $T_{0}>0$, a constant $N\left(T_{0}\right)>0$ and a control $p \in L^{2}\left(0, T_{0}\right)$ such that $Y\left(T_{0} ; y_{0}, p\right)=0$ and

$$
\|p\|_{L^{2}\left(0, T_{0}\right)} \leq N\left(T_{0}\right)\left\|y_{0}\right\| .
$$

Hence, we deduce that the solution of (52) satisfies $y\left(T_{0} ; y_{0}, q\right)=0$ with $q(t)=$ $\mathrm{e}^{\lambda_{j} t} p(t)$. Furthermore,

$$
\begin{aligned}
\|q\|_{L^{2}\left(0, T_{0}\right)}^{2} & =\int_{0}^{T_{0}} \mathrm{e}^{2 \lambda_{j} s}\left|\mathrm{e}^{-\lambda_{j} s} q(s)\right|^{2} \mathrm{~d} s \leq \max \left\{1, \mathrm{e}^{2 \lambda_{j} T_{0}}\right\}\|p\|_{L^{2}\left(0, T_{0}\right)}^{2} \\
& \leq N^{2}\left(T_{0}\right) \max \left\{1, \mathrm{e}^{2 \lambda_{j} T_{0}}\right\}\left\|y_{0}\right\|^{2}
\end{aligned}
$$

We are ready to complete the proof of Theorem 3.7.

Proof of Theorem 3.7 (case $\lambda_{j} \neq 0$ )

Now, in order to deal with the case $\{A, B\} j$-null controllable in time $T_{0}$ and $\lambda_{j} \neq 0$, we consider the operator $A_{j}$ defined in (51).

From Lemma 4.7, we have that $\left\{A_{j}, B\right\}$ is $j$-null controllable in time $T_{0}$. Moreover, the eigenvalues of $A_{j}$ are given by

$$
\mu_{k}=\lambda_{k}-\lambda_{j}, \quad \forall k \in \mathbb{N}^{*}
$$

(in particular, $\mu_{j}=0$ ), with the same eigenfunctions than those of $A,\left\{\varphi_{k}\right\}_{k \in \mathbb{N}^{*}}$.

Observe that $z(t):=\mathrm{e}^{\lambda_{j} t} u(t)$, with $u$ the solution of (11), solves

$$
\left\{\begin{array}{l}
z^{\prime}(t)+A_{j} z(t)+p(t) B z(t)=0, \quad t>0 \\
z(0)=u_{0}
\end{array}\right.
$$


So, we can apply the first part of the proof of Theorem 3.7 (for $\mu_{j}=0$ ) to this problem and deduce that for all $\rho>0$ there exists $R_{\rho}>0$ such that, if $\left\|u_{0}-\varphi_{j}\right\| \leq R_{\rho}$, then

$$
\left\|z(t)-\varphi_{j}\right\| \leq M \mathrm{e}^{-\rho \mathrm{e}^{\omega t}}, \quad \forall t \geq 0,
$$

where $M, \omega$ are positive constants.

Therefore,

$$
\begin{aligned}
\left\|u(t)-\psi_{j}(t)\right\| & =\left\|\mathrm{e}^{-\lambda_{j} t} z(t)-\mathrm{e}^{-\lambda_{j} t} \varphi_{j}\right\| \\
& =\mathrm{e}^{-\lambda_{j} t}\left\|z(t)-\varphi_{j}\right\| \leq M \mathrm{e}^{-\left(\rho \mathrm{e}^{t \omega}+\lambda_{j} t\right)}, \quad \forall t \geq 0 .
\end{aligned}
$$

The conclusion follows by possibly increasing $\rho^{1}$.

\section{Proof of Theorem 3.8}

Let us recall the notion of biorthogonal family and a result we will use to show that $\{A, B\}$ is $j$-null controllable under the conditions of Theorem 3.8.

Definition 5.1. Let $\left\{\zeta_{j}\right\}$ and $\left\{\sigma_{k}\right\}$ be two sequences in a Hilbert space H. We say that the two families are biorthogonal or, that $\left\{\zeta_{j}\right\}$ (resp. $\left\{\sigma_{k}\right\}$ ) is biorthogonal to $\left\{\sigma_{k}\right\}$ (resp. $\left.\left\{\zeta_{j}\right\}\right)$, if

$$
\left\langle\zeta_{j}, \sigma_{k}\right\rangle_{H}=\delta_{j k}, \quad \forall j, k \geq 0
$$

where $\delta_{j k}$ is the Kronecker delta.

The notion of biorthogonal family was used by Fattorini and Russell in [14], where they introduced the moment method. Such a technique was developed later by several authors. We recall below the result proved in [11].

Theorem 5.2. Let $T>0$, let $\left\{\omega_{k}\right\}_{k \in \mathbb{N}}$ be a nonnegative increasing sequence, and set $\zeta_{k}(t):=\mathrm{e}^{\omega_{k} t}, t \in[0, T], k \geq 0$.

If, for some $\alpha>0$,

$$
\sqrt{\omega_{k+1}}-\sqrt{\omega_{k}} \geq \alpha, \quad \forall k \in \mathbb{N}
$$

then there exists a family $\left\{\sigma_{j}\right\}_{j \geq 0}$ which is biorthogonal to $\left\{\zeta_{k}\right\}_{k \geq 0}$ in $L^{2}(0, T)$, that is,

$$
\int_{0}^{T} \sigma_{j}(t) \mathrm{e}^{\omega_{k} t} \mathrm{~d} t=\delta_{j k}, \quad \forall k, j \in \mathbb{N} .
$$

Furthermore, there exist constants $C_{\alpha}, C_{\alpha}(T)>0$ such that

$$
\left\|\sigma_{j}\right\|_{L^{2}(0, T)}^{2} \leq C_{\alpha}^{2}(T) \mathrm{e}^{-2 \omega_{j} T} \mathrm{e}^{C_{\alpha} \sqrt{\omega_{j}} / \alpha}, \quad \forall j \in \mathbb{N} .
$$

\footnotetext{
${ }^{1}$ For $\lambda_{j}>0$, take $\rho=\bar{\rho}$ and for $\lambda_{j}<0$ take $\rho \geq \bar{\rho}+\frac{\left|\lambda_{j}\right|}{\omega}$ Then, $\forall \bar{\rho}>0, \exists R_{\bar{\rho}}>0$ s.t. if $\left\|u_{0}-\varphi_{j}\right\| \leq R_{\bar{\rho}}$, we have that $\left\|u(t)-\psi_{j}(t)\right\| \leq M \mathrm{e}^{-\rho \mathrm{e}^{\bar{\omega} t}}$.
} 
Proof of Theorem 3.8. In order to prove that $\{A, B\}$ is $j$-null controllable, we have to show that there exists $T>0$ such that the following problem

$$
\left\{\begin{array}{l}
y^{\prime}(t)+A y(x)+p(t) B \varphi_{j}=0, \quad t \in[0, T] \\
y(0)=y_{0} \in X
\end{array}\right.
$$

is null controllable with $p \in L^{2}(0, T)$ that satisfies (13).

First, we observe that by the hypothesis on $A$, the eigenfunctions $\left\{\varphi_{k}\right\}_{k \in \mathbb{N}^{*}}$ form an orthonormal basis of $X$.

Let $T>\tau$, where $\tau$ has been defined in (16). For any $y_{0} \in X$ and $p \in L^{2}(0, T)$, it follows from Proposition 4.1 that there exists a unique mild solution $y \in C^{0}([0, T], X)$ of (56) that can be represented by the formula

$$
y(t)=\mathrm{e}^{-t A} y_{0}-\int_{0}^{t} \mathrm{e}^{-(t-s) A} p(s) B \varphi_{j} \mathrm{~d} s .
$$

We want to find $p \in L^{2}(0, T)$ such that $y(T)=0$; thus, the following equality must hold:

$$
\sum_{k \in \mathbb{N}^{*}}\left\langle y_{0}, \varphi_{k}\right\rangle \mathrm{e}^{-\lambda_{k} T} \varphi_{k}=\int_{0}^{T} p(s) \sum_{k \in \mathbb{N}^{*}}\left\langle B \varphi_{j}, \varphi_{k}\right\rangle \mathrm{e}^{-\lambda_{k}(T-s)} \varphi_{k} \mathrm{~d} s .
$$

Therefore,

$$
\left\langle y_{0}, \varphi_{k}\right\rangle=\int_{0}^{T} \mathrm{e}^{\lambda_{k} s} p(s)\left\langle B \varphi_{j}, \varphi_{k}\right\rangle \mathrm{d} s, \quad \forall k \in \mathbb{N}^{*} .
$$

Thus, proving null controllability of the linear system (56) reduces to finding a function $p \in L^{2}(0, T)$ that satisfies

$$
\int_{0}^{T} \mathrm{e}^{\lambda_{k} s} p(s) \mathrm{d} s=\frac{\left\langle y_{0}, \varphi_{k}\right\rangle}{\left\langle B \varphi_{j}, \varphi_{k}\right\rangle}, \quad \forall k \in \mathbb{N}^{*} .
$$

Taking $q(t):=\mathrm{e}^{\lambda_{1} s} p(s)$ and $\omega_{k}:=\lambda_{k}-\lambda_{1} \geq 0$, the family of Eqs. (60) can be rewritten as

$$
\int_{0}^{T} \mathrm{e}^{\omega_{k} s} q(s) \mathrm{d} s=\frac{\left\langle y_{0}, \varphi_{k}\right\rangle}{\left\langle B \varphi_{j}, \varphi_{k}\right\rangle}, \quad \forall k \in \mathbb{N}^{*} .
$$

Thanks to assumption (15), there exists $\alpha>0$ such that the gap condition $\sqrt{\omega_{k+1}}-$ $\sqrt{\omega_{k}} \geq \alpha$ holds for all $k \in \mathbb{N}^{*}$. Then, Theorem 5.2 ensures the existence of a family $\left\{\sigma_{k}\right\}_{k \in \mathbb{N}^{*}}$ such that $\int_{0}^{T} \sigma_{k}(t) \mathrm{e}^{\omega_{j} t} \mathrm{~d} t=\delta_{k j}, \forall k, j \in \mathbb{N}^{*}$.

We claim that the series

$$
\sum_{k \in \mathbb{N}^{*}} \frac{\left\langle y_{0}, \varphi_{k}\right\rangle}{\left\langle B \varphi_{j}, \varphi_{k}\right\rangle} \sigma_{k}(t)
$$


is convergent in $L^{2}(0, T)$. Indeed, appealing to estimate (55) for $\left\{\sigma_{k}\right\}_{k \in \mathbb{N}^{*}}$

$$
\begin{aligned}
\sum_{k \in \mathbb{N}^{*}}\left|\frac{\left\langle y_{0}, \varphi_{k}\right\rangle}{\left\langle B \varphi_{j}, \varphi_{k}\right\rangle}\right|\left\|\sigma_{k}\right\|_{L^{2}(0, T)} & \leq\left\|y_{0}\right\|\left(\sum_{k \in \mathbb{N}^{*}} \frac{\left\|\sigma_{k}\right\|_{L^{2}(0, T)}^{2}}{\left|\left\langle B \varphi_{j}, \varphi_{k}\right\rangle\right|^{2}}\right)^{1 / 2} \\
& \left.\leq\left\|y_{0}\right\|\left(C_{\alpha}^{2}(T) \sum_{k \in \mathbb{N}^{*}} \frac{\mathrm{e}^{-2 \omega_{k} T} \mathrm{e}^{C_{\alpha} \sqrt{\omega_{k}} / \alpha}}{\left|\left\langle B \varphi_{j}, \varphi_{k}\right\rangle\right|^{2}}\right)\right)^{1 / 2} .
\end{aligned}
$$

We observe that the above right-hand side is finite because hypothesis (16) (ii) guarantees

$$
\begin{aligned}
\Lambda_{T} & :=\left(\sum_{k \in \mathbb{N}^{*}} \frac{\mathrm{e}^{-2 \omega_{k} T} \mathrm{e}^{C_{\alpha} \sqrt{\omega_{k}} / \alpha}}{\left|\left\langle B \varphi_{j}, \varphi_{k}\right\rangle\right|^{2}}\right)^{1 / 2} \\
& <C(T, \tau, \alpha)\left(\sum_{k \in \mathbb{N}^{*}} \frac{\mathrm{e}^{-2 \omega_{k} \tau}}{\left|\left\langle B \varphi_{j}, \varphi_{k}\right\rangle\right|^{2}}\right)^{1 / 2}<+\infty
\end{aligned}
$$

because $T>\tau$.

Therefore, the control function $q(t):=\sum_{k \in \mathbb{N}^{*}} \frac{\left\langle y_{0}, \varphi_{k}\right\rangle}{\left\langle B \varphi_{j}, \varphi_{k}\right\rangle} \sigma_{k}(t)$ belongs to $L^{2}(0, T)$ and satisfies

$$
\|q\|_{L^{2}(0, T)} \leq C_{\alpha}(T) \Lambda_{T}\left\|y_{0}\right\|
$$

thanks to the properties of $\left\{\sigma_{k}\right\}_{k \in \mathbb{N}^{*}}$.

Finally, returning to $p$, we deduce that

$$
\|p\|_{L^{2}(0, T)}^{2}=\int_{0}^{T} \mathrm{e}^{-2 \lambda_{1} s}|q(s)|^{2} \mathrm{~d} s \leq \max \left\{1, \mathrm{e}^{-2 \lambda_{1} T}\right\}\|q\|_{L^{2}(0, T)}^{2} .
$$

The conclusion follows by taking

$$
N(T):=\max \left\{1, \mathrm{e}^{\lambda_{1} T}\right\} C_{\alpha}(T) \Lambda_{T} .
$$

\section{Applications}

In this section, we discuss examples of bilinear control systems to which we can apply Corollary 3.10. The first problems we study are $1 \mathrm{D}$ parabolic equations of the form

$$
u_{t}(t, x)-u_{x x}(t, x)+p(t) B u(t, x)=0, \quad(t, x) \in[0, T] \times(0,1)
$$

in the state space $X=L^{2}(0,1)$, with Dirichlet or Neumann boundary conditions and with $B$ the following multiplication operators:

$$
B u(t, x)=\mu(x) u(t, x),
$$


where the functions $\mu$ are in some appropriate Sobolev space.

Then, we prove the superexponential stabilizability of the following one-dimensional equation with variable coefficients

$$
u_{t}(t, x)-\left((1+x)^{2} u_{x}(t, x)\right)_{x}+p(t) B u(t, x)=0
$$

with Dirichlet boundary conditions.

Finally, we apply Corollary 3.10 to the following parabolic equation

$$
u_{t}(t, x)-\Delta u(t, x)+p(t) B u(t, x)=0, \quad(t, x) \in[0, T] \times B^{3}
$$

for radial data in the 3D unit ball $B^{3}$.

In each example, we will denote by $\left\{\lambda_{k}\right\}_{k \in \mathbb{N}^{*}}$ and $\left\{\varphi_{k}\right\}_{k \in \mathbb{N}^{*}}$, respectively, the eigenvalues and eigenfunctions of the second-order operator associated with the problem under investigation. We will take $(\bar{u}, \bar{p})=\left(\psi_{j}, 0\right)$ as reference trajectory-control pair, where $\psi_{j}=\mathrm{e}^{-\lambda_{j} t} \varphi_{j}$ are the eigensolutions associated with the second-order operator at hand.

Remark 6.1. All the second-order operators considered in the aforementioned examples are accretive, that is, $\langle A x, x\rangle \geq 0$, for all $x \in D(A)$. Hence, the eigenvalues $\left\{\lambda_{k}\right\}_{k \in \mathbb{N}^{*}}$ are nonnegative.

In this case, we claim that it is sufficient to verify the following gap condition to have (15):

$$
\exists \alpha>0: \sqrt{\lambda_{k+1}}-\sqrt{\lambda_{k}} \geq \alpha, \quad \forall k \geq 1
$$

Indeed, if (66) holds, we have that

$$
\begin{aligned}
\sqrt{\lambda_{k+1}-\lambda_{1}}-\sqrt{\lambda_{k}-\lambda_{1}} & =\frac{\lambda_{k+1}-\lambda_{k}}{\sqrt{\lambda_{k+1}-\lambda_{1}}+\sqrt{\lambda_{k}-\lambda_{1}}} \\
& \geq \frac{\lambda_{k+1}-\lambda_{k}}{\sqrt{\lambda_{k+1}}+\sqrt{\lambda_{k}}}=\sqrt{\lambda_{k+1}}-\sqrt{\lambda_{k}} \geq \alpha,
\end{aligned}
$$

for all $k \geq 1$, that is (15).

\subsection{Dirichlet boundary conditions}

Let $\Omega=(0,1), X=L^{2}(\Omega)$ and consider the problem

$$
\begin{cases}u_{t}(t, x)-u_{x x}(t, x)+p(t) \mu(x) u(t, x)=0 & x \in \Omega, t>0 \\ u=0 & x \in \partial \Omega, t>0 \\ u(0, x)=u_{0}(x) & x \in \Omega,\end{cases}
$$

where $p \in L^{2}(0, T)$ is the control function, $u$ the state variable, and $\mu$ is a function in $H^{3}(\Omega)$.

We denote by $A$ the operator defined by

$$
D(A)=H^{2} \cap H_{0}^{1}(\Omega), \quad A \varphi=-\frac{d^{2} \varphi}{d x^{2}} .
$$


$A$ satisfies all the properties in (6): In particular, it is strictly accretive and its eigenvalues and eigenfunctions have the following explicit expressions:

$$
\lambda_{k}=(k \pi)^{2}, \quad \varphi_{k}(x)=\sqrt{2} \sin (k \pi x), \quad \forall k \in \mathbb{N}^{*} .
$$

It is straightforward to prove that the eigenvalues fulfill the required gap property. Indeed,

$$
\sqrt{\lambda_{k+1}}-\sqrt{\lambda_{k}}=(k+1) \pi-k \pi=\pi, \quad \forall k \in \mathbb{N}^{*} .
$$

So, (66) is satisfied (hence (15) holds).

In order to apply Corollary 3.10 to system (67) and deduce the superexponential stabilizability to the trajectory $\psi_{j}$, we need to prove that

- $\left\langle B \varphi_{j}, \varphi_{k}\right\rangle \neq 0$, for all $k \in \mathbb{N}^{*}$,

- there exists $\tau>0$ such that

$$
\sum_{k \in \mathbb{N}^{*}} \frac{\mathrm{e}^{-2 \lambda_{k} \tau}}{\left|\left\langle B \varphi_{j}, \varphi_{k}\right\rangle\right|^{2}}<+\infty
$$

Following the strategy in $[5$, formula $(8)]$ (for the case $j=1$ ), we find that

$$
\left\langle\mu \varphi_{j}, \varphi_{k}\right\rangle=\frac{4}{k^{3} \pi^{2}}\left[\mu^{\prime}(1)(-1)^{k+j}-\mu^{\prime}(0)\right]-\frac{\sqrt{2}}{(k \pi)^{3}} \int_{0}^{1}\left(\mu \varphi_{j}\right)^{\prime \prime \prime}(x) \cos (k \pi x) \mathrm{d} x
$$

Thus, if

$$
\mu^{\prime}(1) \pm \mu^{\prime}(0) \neq 0 \quad \text { and } \quad\left\langle\mu \varphi_{j}, \varphi_{k}\right\rangle \neq 0, \quad \forall k \in \mathbb{N}^{*}
$$

we deduce that $\left\langle\mu \varphi_{j}, \varphi_{k}\right\rangle$ is of order $1 / k^{3}$ as $k \rightarrow \infty$.

Remark 6.2. An example of a function which satisfies (69) is $\mu(x)=x^{2}$. Indeed, in this case

$$
\left\langle\mu \varphi_{j}, \varphi_{k}\right\rangle= \begin{cases}\frac{4 k j(-1)^{k+j}}{\left(k^{2}-j^{2}\right)^{2}}, & k \neq j, \\ \frac{2 j^{2} \pi^{2}-3}{6 j^{2} \pi^{2}}, & k=j,\end{cases}
$$

and so $\left\langle\mu \varphi_{j}, \varphi_{k}\right\rangle \neq 0$ for all $k \in \mathbb{N}^{*}$ and furthermore there exists a constant $C_{j}>0$, that depends on $j$, such that

$$
\left|\left\langle\mu \varphi_{j}, \varphi_{k}\right\rangle\right| \geq \frac{C_{j}}{\lambda_{k}^{3 / 2}}, \quad \forall k \in \mathbb{N}^{*} .
$$

We conclude that, under assumption (69),

$$
\exists C>0 \text { such that }\left|\left\langle B \varphi_{j}, \varphi_{k}\right\rangle\right| \geq C k^{-3}=C \lambda_{k}^{-3 / 2}, \quad \forall k \in \mathbb{N}^{*},
$$

and thanks to the polynomial behavior of the bound

$$
\sum_{k \in \mathbb{N}^{*}} \frac{\mathrm{e}^{-2 \lambda_{k} \tau}}{\left|\left\langle B \varphi_{j}, \varphi_{k}\right\rangle\right|^{2}}<\infty
$$


for all $\tau>0$.

Therefore, all the hypotheses of Corollary 3.10 are satisfied and system (67), with initial condition $u_{0}$ in a neighborhood of the eigenstate $\varphi_{j}$, is superexponentially stabilizable to the eigensolution $\psi_{j}$, for all $j \geq 1$.

Remark 6.3. Assumption (70) for problem (67) is not too restrictive. In fact, it is possible to prove that the set of functions in $H^{3}(\Omega)$ for which (70) holds is dense in $H^{3}(\Omega)$. For a proof of this fact, see Appendix A in [5].

\subsection{Neumann boundary conditions}

Now, we look at an example with Neumann boundary conditions: Let $\Omega=(0,1)$ and consider the following bilinear stabilizability problem:

$$
\begin{cases}u_{t}(t, x)-\partial_{x}^{2} u(t, x)+p(t) \mu(x) u(t, x)=0 & x \in \Omega, t>0 \\ u_{x}=0 & x \in \partial \Omega, t>0 \\ u(0, x)=u_{0}(x) & x \in \Omega\end{cases}
$$

Let $X=L^{2}(\Omega)$. When we rewrite (71) in abstract form, the operators $A$ and $B$ are defined by

$$
\begin{aligned}
& D(A)=\left\{\varphi \in H^{2}(0,1): \varphi^{\prime}=0 \text { on } \partial \Omega\right\}, \quad A \varphi=-\frac{d^{2} \varphi}{d x^{2}} \\
& D(B)=X, \quad B \varphi=\mu \varphi .
\end{aligned}
$$

where $\mu$ is a real-valued function in $H^{2}(\Omega)$.

Operator $A$ satisfies the assumptions in (6), and it is possible to compute explicitly its eigenvalues and eigenfunctions:

$$
\begin{aligned}
& \lambda_{0}=0, \quad \varphi_{0}=1 \\
& \lambda_{k}=(k \pi)^{2}, \varphi_{k}(x)=\sqrt{2} \cos (k \pi x), \quad \forall k \geq 1 .
\end{aligned}
$$

Since the eigenvalues are the same of those in Example 6.1 for $k \geq 1$, the gap condition is satisfied for all $k \geq 0$.

Integrating by parts, we find that the scalar product $\left\langle\mu \varphi_{j}, \varphi_{k}\right\rangle$ is equal to

$$
\left\langle\mu \varphi_{j}, \varphi_{k}\right\rangle= \begin{cases}\frac{\sqrt{2}}{(k \pi)^{2}}\left(\mu^{\prime}(1)(-1)^{k}-\mu^{\prime}(0)\right)-\frac{\sqrt{2}}{(k \pi)^{2}} \int_{0}^{1} \mu^{\prime \prime}(x) \cos (k \pi x) \mathrm{d} x, & j=0, \\ \frac{2}{(k \pi)^{2}}\left(\mu^{\prime}(1)(-1)^{k+j}-\mu^{\prime}(0)\right)-\frac{2}{(k \pi)^{2}} \int_{0}^{1}\left(\mu(x) \varphi_{j}(x)\right)^{\prime \prime} \cos (k \pi x) \mathrm{d} x, & j \geq 1 .\end{cases}
$$

Thus, reasoning as Example 6.1, if $\left\langle B \varphi_{j}, \varphi_{k}\right\rangle \neq 0, \forall k \in \mathbb{N}$ and $\mu^{\prime}(1) \pm \mu^{\prime}(0) \neq 0$, we have that

$$
\exists C>0 \text { such that }\left|\left\langle B \varphi_{j}, \varphi_{k}\right\rangle\right| \geq C k^{-2}=C \lambda_{k}^{-1}, \quad \forall k \in \mathbb{N}^{*},
$$

and therefore the series in (16) is finite for all $\tau>0$. 
Remark 6.4. An example of a suitable function $\mu$ for problem (71) that satisfies the above hypothesis is $\mu(x)=x^{2}$, for which

$$
\left\langle\mu \varphi_{0}, \varphi_{k}\right\rangle= \begin{cases}\frac{2 \sqrt{2}(-1)^{k}}{(k \pi)^{2}}, & k \geq 1 \\ \frac{1}{3}, & k=0\end{cases}
$$

and for $j \neq 0$

$$
\left\langle\mu \varphi_{j}, \varphi_{k}\right\rangle= \begin{cases}\frac{4(-1)^{k+j}\left(k^{2}+j^{2}\right)}{\left(k^{2}-j^{2}\right)^{2} \pi^{2}}, & k \neq j, \\ \frac{1}{3}+\frac{1}{2 j^{2} \pi^{2}}, & k=j .\end{cases}
$$

Applying Corollary 3.10, it follows that system (71), with initial condition $u_{0}$ in a neighborhood of $\varphi_{j}$, is superexponentially stabilizable to the eigensolution $\psi_{j}$, for any $j \in \mathbb{N}$.

6.3. Dirichlet boundary conditions, variable coefficients

In this example, we analyze the superexponential stabilizability of a parabolic equation in divergence form with nonconstant coefficients in the second-order term.

Let $\Omega=(0,1), X=L^{2}(\Omega)$ and consider the problem

$$
\begin{cases}u_{t}(t, x)-\left((1+x)^{2} u_{x}(t, x)\right)_{x}+p(t) \mu(x) u(t, x)=0 & x \in \Omega, t>0 \\ u(t, 0)=0, \quad u(t, 1)=0, & t>0 \\ u(0, x)=u_{0}(x) & x \in \Omega\end{cases}
$$

where $p \in L^{2}(0, T)$ is the control and $\mu$ is a function in $H^{2}(\Omega)$ with some properties to be specified later.

We denote by $A$ the operator

$$
A: D(A) \subset X \rightarrow X, \quad A u=-\left((1+x)^{2} u_{x}\right)_{x}
$$

where $D(A)=H^{2} \cap H_{0}^{1}(\Omega)$ and it is possible to prove that $A$ satisfies the properties in (6). The eigenvalues and eigenfunctions of $A$ are computed as follows:

$$
\lambda_{k}=\frac{1}{4}+\left(\frac{k \pi}{\ln 2}\right)^{2}, \quad \varphi_{k}(x)=\sqrt{\frac{2}{\ln 2}}(1+x)^{-1 / 2} \sin \left(\frac{k \pi}{\ln 2} \ln (1+x)\right), \quad \forall k \in \mathbb{N}^{*} .
$$

The gap condition (66) holds:

$$
\sqrt{\lambda_{k+1}}-\sqrt{\lambda_{k}} \geq \frac{\pi}{\ln 2}, \quad \forall k \in \mathbb{N}^{*} .
$$

Hence, from Remark 6.1, hypothesis (15) is verified.

Now, we check the hypotheses on the operator $B \varphi=\mu \varphi$ needed to apply Corollary 3.10. We recall that we want to prove that: 
- $\left\langle B \varphi_{j}, \varphi_{k}\right\rangle \neq 0$, for all $k \in \mathbb{N}^{*}$,

- there exists $\tau>0$ such that

$$
\sum_{k \in \mathbb{N}^{*}} \frac{\mathrm{e}^{-2 \lambda_{k} \tau}}{\left.\left\langle B \varphi_{j}, \varphi_{k}\right\rangle\right|^{2}}<+\infty
$$

Let us compute the Fourier coefficients of $B \varphi_{j}$ :

$$
\begin{aligned}
\left\langle\mu \varphi_{j}, \varphi_{k}\right\rangle= & \sqrt{\frac{2}{\ln 2}} \int_{0}^{1} \mu(x) \varphi_{j}(x)(1+x)^{-1 / 2} \sin \left(\frac{k \pi}{\ln 2} \ln (1+x)\right) \mathrm{d} x \\
= & \sqrt{\frac{2}{\ln 2}} \frac{\ln 2}{k \pi}\left(-\left.\mu(x) \varphi_{j}(x)(1+x)^{1 / 2} \cos \left(\frac{k \pi}{\ln 2} \ln (1+x)\right)\right|_{0} ^{1}\right. \\
& \left.+\int_{0}^{1}\left(\mu(x) \varphi_{j}(x)(1+x)^{1 / 2}\right)^{\prime} \cos \left(\frac{k \pi}{\ln 2} \ln (1+x)\right) \mathrm{d} x\right) \\
= & \sqrt{\frac{2}{\ln 2}}\left(\frac{\ln 2}{k \pi}\right)^{2}\left(\left.\left(\mu(x) \varphi_{j}(x)(1+x)^{1 / 2}\right)^{\prime}(1+x) \sin \left(\frac{k \pi}{\ln 2} \ln (1+x)\right)\right|_{0} ^{1}\right. \\
& \left.-\int_{0}^{1}\left(\left(\mu(x) \varphi_{j}(x)(1+x)^{1 / 2}\right)^{\prime}(1+x)\right)^{\prime} \sin \left(\frac{k \pi}{\ln 2} \ln (1+x)\right) \mathrm{d} x\right) \\
= & \sqrt{\frac{2}{\ln 2}}\left(\frac{\ln 2}{k \pi}\right)^{3}\left(\left.\left(\left(\mu(x) \varphi_{j}(x)(1+x)^{1 / 2}\right)^{\prime}(1+x)\right)^{\prime}(1+x) \cos \left(\frac{k \pi}{\ln 2} \ln (1+x)\right)\right|_{0} ^{1}\right. \\
& \left.-\int_{0}^{1}\left(\left(\left(\mu(x) \varphi_{j}(x)(1+x)^{1 / 2}\right)^{\prime}(1+x)\right)^{\prime}(1+x)\right)^{\prime} \cos \left(\frac{k \pi}{\ln 2} \ln (1+x)\right) \mathrm{d} x\right) \\
= & \sqrt{\frac{2}{\ln 2}}\left(\frac{\ln 2}{k \pi}\right)^{3}\left(\sqrt{\frac{2}{\ln 2}} \frac{2 j \pi}{\ln 2}\left(2 \mu^{\prime}(1)(-1)^{k+j}-\mu^{\prime}(0)\right)\right. \\
& \left.-\int_{0}^{1}\left(\left(\left(\mu(x) \varphi_{j}(x)(1+x)^{1 / 2}\right)^{\prime}(1+x)\right)^{\prime}(1+x)\right)^{\prime} \cos \left(\frac{k \pi}{\ln 2} \ln (1+x)\right) \mathrm{d} x\right)
\end{aligned}
$$

Observe that, for the same reason of Example 6.1, if $2 \mu^{\prime}(1) \pm \mu^{\prime}(0) \neq 0$ and $\left\langle\mu \varphi_{j}, \varphi_{k}\right\rangle \neq 0, \forall k \in \mathbb{N}^{*}$, then there exists a constant $C>0$ such that $\left|\left\langle B \varphi_{j}, \varphi_{k}\right\rangle\right|$ is bounded from below by $C \lambda_{k}^{-3 / 2}$, for all $k \in \mathbb{N}^{*}$. Thus, series (74) is finite for all $\tau>0$.

Remark 6.5. Let us fix $j=1$. As an example of a function $\mu$ that verifies the lower bound $\left|\left\langle\mu \varphi_{1}, \varphi_{k}\right\rangle\right| \geq C \lambda_{k}^{-3 / 2}$, one can consider again $\mu(x)=x$ : Indeed, it satisfies the sufficient condition $2 \mu^{\prime}(1) \pm \mu^{\prime}(0) \neq 0$ and the Fourier coefficients of $\left(B \varphi_{1}\right)(x)=$ $x \varphi_{1}(x)$ are all different from zero:

$$
\begin{aligned}
& \left\langle\mu \varphi_{1}, \varphi_{k}\right\rangle= \\
& \quad= \begin{cases}\frac{2\left(2(-1)^{k+1}-1\right)}{\left(k^{2}-1\right)^{2}\left(1+\frac{(k+1)^{2} \pi^{2}}{(\ln 2)^{2}}\right)\left(1+\frac{(k-1)^{2} \pi^{2}}{(\ln 2)^{2}}\right)}\left(4 k^{3}+k+1+2 k\left(k^{2}-1\right)^{2} \frac{\pi}{(\ln 2)^{2}}\right), & k \geq 2 \\
\frac{1}{\ln 2}\left(\frac{(1-\ln 2)\left(\frac{2 \pi}{\ln 2}\right)^{3}-\frac{2 \pi}{\ln 2}}{1+\left(\frac{2 \pi}{\ln 2}\right)^{3}}\right), & k=1\end{cases}
\end{aligned}
$$

This concludes the verification of the hypotheses of Corollary 3.10 that imply the superexponential stabilizability of (73) with initial condition $u_{0}$ in a neighborhood of $\varphi_{j}$ to the eigensolution $\psi_{j}$, for all $j \in \mathbb{N}^{*}$. 


\section{4. $3 D$ ball with radial data}

In this example, we consider an evolution equation in the three-dimensional unit ball $B^{3}$ for radial data. The bilinear stabilizability problem is the following:

$$
\begin{cases}u_{t}(t, r)-\Delta u(t, r)+p(t) \mu(r) u(t, r)=0 & r \in[0,1], t>0 \\ u(t, 1)=0, & t>0 \\ u(0, r)=u_{0}(r) & r \in[0,1]\end{cases}
$$

where the Laplacian in polar coordinates for radial data has the form

$$
\Delta \varphi(r)=\partial_{r}^{2} \varphi(r)+\frac{2}{r} \partial_{r} \varphi(r) .
$$

The function $\mu$ is a radial function as well in the space $H_{r}^{3}\left(B^{3}\right)$, where the spaces $H_{r}^{k}\left(B^{3}\right)$ are defined as follows:

$$
\begin{aligned}
X & :=L_{r}^{2}\left(B^{3}\right)=\left\{\varphi \in L^{2}\left(B^{3}\right) \mid \exists \psi: \mathbb{R} \rightarrow \mathbb{R}, \varphi(x)=\psi(|x|)\right\} \\
H_{r}^{k}\left(B^{3}\right) & :=H^{k}\left(B^{3}\right) \cap L_{r}^{2}\left(B^{3}\right) .
\end{aligned}
$$

The domain of the Dirichlet Laplacian $A:=-\Delta$ in $X$ is $D(A)=H_{r}^{2} \cap H_{0}^{1}\left(B^{3}\right)$. We observe that $A$ satisfies the hypotheses required to apply Theorem 3.10. We denote by $\left\{\lambda_{k}\right\}_{k \in \mathbb{N}^{*}}$ and $\left\{\varphi_{k}\right\}_{k \in \mathbb{N}^{*}}$ the families of eigenvalues and eigenfunctions of $A, A \varphi_{k}=$ $\lambda_{k} \varphi_{k}$, namely

$$
\lambda_{k}=(k \pi)^{2}, \quad \varphi_{k}=\frac{\sin (k \pi r)}{\sqrt{2 \pi} r}, \quad \forall k \in \mathbb{N}^{*} .
$$

See [21], section 8.14. The family $\left\{\varphi_{k}\right\}_{k \in \mathbb{N}^{*}}$ forms an orthonormal basis of $X$.

In order to prove a superexponential stabilizability result to the eigensolutions $\psi_{j}$, we need to verify the remaining hypotheses in Corollary 3.10 regarding the gap condition of the eigenvalues of $A$ and the properties of the operator $B: X \mapsto X, B \varphi=\mu \varphi$.

Since the Laplacian in the $3 D$ ball for radial data behaves as a one-dimensional operator, the analysis is very similar to the previous cases. Indeed, since the eigenvalues of the operator $A$ are actually the same of the $1 D$ Dirichlet Laplacian, we have

$$
\sqrt{\lambda_{k+1}}-\sqrt{\lambda_{k}}=\pi, \quad \forall k \in \mathbb{N}^{*} .
$$

In order to compute a suitable lower bound for the Fourier coefficients of $B \varphi_{j}$, we recall the following property of radial symmetric functions $f=f(r)$ : The integral over the unit ball $B^{n} \subset \mathbb{R}^{n}$ of $f=f(r)$ reduces to

$$
\int_{B^{n}} f \mathrm{~d} V=\left|S^{n-1}\right| \int_{0}^{1} f(r) r^{n-1} \mathrm{~d} r
$$

where $\left|S^{n-1}\right|$ is the measure of the surface of the sphere $S^{n-1}$. 
Therefore,

$$
\begin{aligned}
\left\langle\mu \varphi_{j}, \varphi_{k}\right\rangle= & \int_{B^{3}} \frac{1}{2 \pi} \mu(r) \frac{\sin (j \pi r)}{r} \frac{\sin (k \pi r)}{r} \mathrm{~d} V \\
= & 4 \pi \int_{0}^{1} \frac{1}{2 \pi} \mu(r) \frac{\sin (j \pi r)}{r} \frac{\sin (k \pi r)}{r} r^{2} \mathrm{~d} r \\
= & \int_{0}^{1} 2 \mu(r) \sin (j \pi r) \sin (k \pi r) \mathrm{d} r \\
= & \frac{4 j}{k^{3} \pi^{2}}\left(\mu^{\prime}(1)(-1)^{k+j}-\mu^{\prime}(0)\right)+ \\
& -\frac{2}{(k \pi)^{3}} \int_{0}^{1}(\mu(r) \sin (j \pi r))^{\prime \prime \prime} \cos (k \pi r) \mathrm{d} r .
\end{aligned}
$$

Following the same argument as in Example 6.1, if all the coefficients $\left\langle\mu \varphi_{j}, \varphi_{k}\right\rangle$ are different from zero and, moreover, $\mu^{\prime}(1) \pm \mu^{\prime}(0) \neq 0$, then there exists a constant $C>0$ such that

$$
\left|\left\langle\mu \varphi_{j}, \varphi_{k}\right\rangle\right| \geq C \lambda_{k}^{-3 / 2}, \quad \forall k \in \mathbb{N}^{*}
$$

and thus the series in (74) is finite also in this case, for all $\tau>0$.

Remark 6.6. An example of a function $\mu \in H_{r}^{3}\left(B^{3}\right)$ with the aforementioned properties is $\mu(r)=r^{2}$. In this case, the Fourier coefficients of $B \varphi_{j}$ are defined by

$$
\left\langle B \varphi_{j}, \varphi_{k}\right\rangle= \begin{cases}\frac{4(-1)^{k+j} k j}{\left(k^{2}-j^{2}\right)^{2} \pi^{2}}, & k \neq j, \\ \frac{2 j^{2} \pi^{2}-3}{6 j^{2} \pi^{2}}, & k=j .\end{cases}
$$

Finally, applying Corollary 3.10, we deduce that, fixed $T>0$, for all $\rho>0$, there exists $R_{\rho}>0$ such that, if the initial condition $u_{0}$ satisfies $\left\|u_{0}-\varphi_{j}\right\| \leq R_{\rho}$, then

$$
\left\|u(t)-\psi_{j}(t)\right\| \leq M \mathrm{e}^{-\rho \mathrm{e}^{\omega t}}, \quad \forall t>0
$$

where $M, \omega>0$ are suitable constants.

\section{Acknowledgements}

The authors would like to thank K. Beauchard for her precious comments and the anonymous reviewers for their suggestions which helped us to improve our manuscript. The last two authors would also like to thank A. Duca for interesting discussions.

Funding Open access funding provided by Università degli Studi di Roma Tor Vergata within the CRUICARE Agreement. 
Open Access. This article is licensed under a Creative Commons Attribution 4.0 International License, which permits use, sharing, adaptation, distribution and reproduction in any medium or format, as long as you give appropriate credit to the original author(s) and the source, provide a link to the Creative Commons licence, and indicate if changes were made. The images or other third party material in this article are included in the article's Creative Commons licence, unless indicated otherwise in a credit line to the material. If material is not included in the article's Creative Commons licence and your intended use is not permitted by statutory regulation or exceeds the permitted use, you will need to obtain permission directly from the copyright holder. To view a copy of this licence, visit http://creativecommons.org/licenses/ by/4.0/.

Publisher's Note Springer Nature remains neutral with regard to jurisdictional claims in published maps and institutional affiliations.

\section{REFERENCES}

[1] F. Alabau-Boussouira, P. Cannarsa, and C. Urbani. Exact controllability to the ground state solution for evolution equations of parabolic type via bilinear control. preprint available on arXiv:1811.08806.

[2] F. Alabau-Boussouira, P. Cannarsa, and C. Urbani. Bilinear control for evolution equations of parabolic type with unbounded lower order terms. in preparation.

[3] J.M. Ball, J.E. Marsden, and M. Slemrod. Controllability for distributed bilinear systems. SIAM Journal on Control and Optimization, 20(4):575-597, 1982.

[4] K. Beauchard. Local controllability and non-controllability for a $1 \mathrm{~d}$ wave equation with bilinear control. Journal of Differential Equations, 250(4):2064-2098, 2011.

[5] K. Beauchard and C. Laurent. Local controllability of 1d linear and nonlinear Schrödinger equations with bilinear control. J. Math. Pures Appl., 94:520-554, 2010.

[6] K. Beauchard and M. Morancey. Local controllability of 1D Schrödinger equations with bilinear control and minimal time. Mathematical Control \& Related Fields, 4(2):125-160, 2014.

[7] A. Bensoussan, G. Da Prato, M.C. Delfour, and S.K. Mitter. Representation and control of infinite dimensional systems, volume 1. Birkhäuser Boston, 1992.

[8] P. Cannarsa, G. Floridia, and A. Y. Khapalov. Multiplicative controllability for semilinear reactiondiffusion equations with finitely many changes of sign. Journal de Mathématiques Pures et Appliquées, 108(4):425-458, 2017.

[9] P. Cannarsa and A. Y. Khapalov. Micromotions and controllability of a swimming model in an incompressible fluid governed by 2-d or 3-d navier-stokes equations. Journal of Mathematical Analysis and Applications, 465(1):100-124, 2018.

[10] P. Cannarsa and A.Y. Khapalov. Multiplicative controllability for reaction-diffusion equations with target states admitting finitely many changes of sign. Discrete Contin. Dyn. Syst. Ser. B, 14:12931311, 2010.

[11] P. Cannarsa, P. Martinez, and J. Vancostenoble. The cost of controlling weakly degenerate parabolic equations by boundary controls. Mathematical Control \& Related Fields, 7(2):171-211, 2017.

[12] P. Cannarsa and C. Urbani. Superexponential stabilizability of degenerate parabolic equations via bilinear control. Inverse Problems and Related Topics, 310:31-45, 2020.

[13] T. Chambrion, P. Mason, M. Sigalotti, and U. Boscain. Controllability of the discrete-spectrum Schrödinger equation driven by an external field. Annales de l'IHP Analyse non linéaire, 26(1):329_ 349, 2009.

[14] H.O. Fattorini and D.L. Russell. Exact controllability theorems for linear parabolic equations in one space dimension. Archive for Rational Mechanics and Analysis, 43(4):272-292, 1971.

[15] E. Fernández-Cara, S. Guerrero, O Y. Imanuvilov, and J.-P. Puel. Local exact controllability of the Navier-Stokes system. Journal de Mathématiques Pures et Appliquées, 83(12):1501-1542, 2004.

[16] G. Floridia. Approximate controllability for nonlinear degenerate parabolic problems with bilinear control. Journal of Differential Equations, 257(9):3382-3422, 2014. 
[17] A. Khapalov, P. Cannarsa, F. S. Priuli, and G. Floridia. Well-posedness of 2-d and 3-d swimming models in incompressible fluids governed by Navier-Stokes equations. Journal of Mathematical Analysis and Applications, 429(2):1059-1085, 2015.

[18] A.Y. Khapalov. Global non-negative controllability of the semilinear parabolic equation governed by bilinear control. ESAIM: Control, Optimisation and Calculus of Variations, 7:269-283, 2002.

[19] A.Y. Khapalov. On bilinear controllability of the parabolic equation with the reaction-diffusion term satisfying newton's law. J. Comput. Appl. Math, 21(1):275-297, 2002.

[20] A.Y. Khapalov. Controllability of partial differential equations governed by multiplicative controls. Springer, Berlin, 2010.

[21] N.N. Lebedev. Special functions and their applications. Revised English edition. Translated and edited by Richard A. Silverman. Prentice-Hall Inc., Englewood Cliffs, NJ, 1965.

[22] J.-L. Lions. Contrôlabilité exacte, perturbations et stabilisation de systèmes distribués. tome 1. RMA, 8, 1988.

[23] J.L. Lions. Contrôlabilité exacte, stabilisation et perturbations de systemes distribues. tome 2., 1988.

Fatiha Alabau-Boussouira and Cristina Urbani

Laboratoire Jacques-Louis Lions Sorbonne Université

75005 Paris

France

E-mail:alabau@ljll.math.upmc.fr

Cristina Urbani

E-mail: cristina.urbani@gssi.it

Piermarco Cannarsa

Dipartimento di Matematica

Università di Roma Tor Vergata

00133 Rome

Italy

E-mail: cannarsa@mat.uniroma2.it

Cristina Urbani

Gran Sasso Science Institute and Sorbonne Université 67100 L'Aquila

Italy

Fatiha Alabau-Boussouira

Université de Lorraine

$57000 \mathrm{Metz}$

France 